\title{
Deterministic extinction by mixing in cyclically competing species
}

\author{
Cilie W. Feldager, ${ }^{1}$ Namiko Mitarai,,${ }^{1,}$ * and Hiroki Ohta ${ }^{2, \dagger}$ \\ 1 Center for Models of Life, Niels Bohr Institute, \\ University of Copenhagen, Blegdamsvej 17, 2100 Copenhagen, Denmark. \\ ${ }^{2}$ Niels Bohr International Academy / Center for Models of Life, Niels Bohr Institute, \\ University of Copenhagen, Blegdamsvej 17, 2100 Copenhagen, Denmark.
}

(Dated: June 14, 2021)

\begin{abstract}
We consider a cyclically competing species model on a ring with global mixing at finite rate, which corresponds to the well-known Lotka-Volterra equation in the limit of infinite mixing rate. Within a perturbation analysis of the model from the infinite mixing rate, we provide analytical evidence that extinction occurs deterministically at sufficiently large but finite values of the mixing rate for any species number $N \geq 3$. Further, by focusing on the cases of rather small species numbers, we discuss numerical results concerning the trajectories toward such deterministic extinction, including global bifurcations caused by changing the mixing rate.
\end{abstract}

PACS numbers: 87.23.Cc, 05.50.+q, 05.10.Gg

\section{INTRODUCTION}

One of the central interests in the field of theoretical ecology and game theory is to understand the mechanism of coexistence and extinction in interacting agents [1]3. Among many kinds of models for interacting agents, $N$-species cyclic dominance has been studied intensely due to rich dynamics and possible long-term coexistence. Specifically, simple models for such cyclic dominance have been introduced based on differential equations [4, 5] or lattice models 6 6], and could provide a fundamental viewpoint to understand more complicated cases observed in nature $9-12$.

Dynamical behaviors of cyclic dominance between interacting agents may be described by the Lotka-Volterra (LV) equation or a cyclically competing species model on a lattice, where the former is supposed to be derived in the large size limit of the latter in a well-mixed condition. Specifically, the time evolution of the density $P_{\alpha}$ of a species $\alpha$ for $\alpha \in\{1,2, \cdots, N\}$ in the $\mathrm{LV}$ equation is described as follows [1]:

$$
\dot{P}_{\alpha}=P_{\alpha}\left(P_{\alpha_{+}}-P_{\alpha_{-}}\right),
$$

where $\dot{P}_{\alpha}$ is the time derivative of $P_{\alpha} \cdot \alpha_{+}\left(\alpha_{-}\right)$denotes a species forward (backward) next to $\alpha$ in the cyclic sense, i.e., it is a prey (predator) of species $\alpha$ (Fig. 1 left panel).

One of the characteristic properties in the LV equation is the coexistence of all the species where each density shows neutrally stable oscillations with an amplitude given by the initial condition [1]. The other characteristic properties in LV equation often discussed are qualitative differences between the odd and even species number cases [2, 13]. For example, in the even species number case, odd-labeled species and even-labeled species antagonize each other, while in the odd species number case, the overall interaction forms a negative feedback loop. There has been still recent progress on analytical topics in the LV equation, focusing on conserved quantities

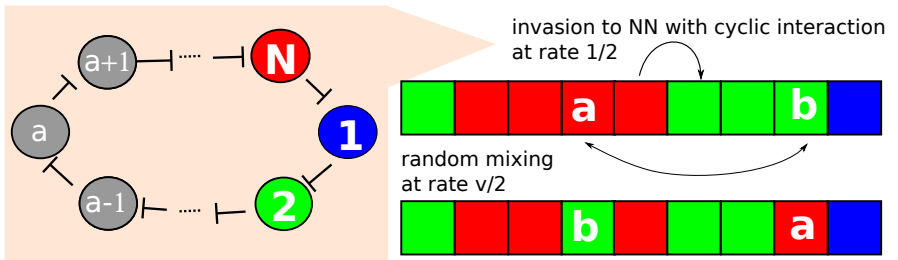

FIG. 1: (color online) A schematic description of the model. An invasion through the nearest neighbor (NN) occurs along the cyclic competition. Individual species on randomly chosen two sites are exchanged at rate $v / 2$.

[14, 15] or the related Lyapunov functions in the case of more general heterogeneous interactions [16 18].

On the other hand, a cyclically competing species model on a lattice, where each species on a lattice stochastically invades its neighbor species along the rule of the cyclic dominance, shows different behaviors from those in the LV equation even qualitatively. For example, in the one-dimensional case with the infinite system size, it has been proven that when $N \leq 4$, species at any site is invaded after any time period, showing slow coarsening, while when $N \geq 5$, each site reaches a final state, leading to an absorbing state [7, 19,21]. Although it has been poorly known how the behaviors of models depend on the dimensionality of the lattices, an approximation analysis rather suggests that the behaviors of the models do not approach those in the LV equation even if the spatial dimension is higher 22 24.

As mentioned above, mixing in lattice models is supposed to bridge the gap between the two descriptions of cyclic ecosystems. However, if the system is not completely well-mixed, it could generally produce some macroscopic behaviors qualitatively different from those in the well-mixed condition. For example, one might consider mixing by local mobility, i.e., by locally exchanging the position of two species or mixing induced by a fluid flow, resulting in spatiotemporal density oscillations 
such as spirals [25, 26] or density oscillations with an increasing amplitude leading to extinction [27, respectively. Thus, such numerical works have already shown that mixing tends to induce oscillatory behaviors. However, how a mixed cyclic ecosystem approaches the LV equation is still not entirely clear. In this paper, we propose a simple model where one can extract analytical results, to some extent, on this topic.

In this paper, we study a cyclically competing species model on a ring by taking into account the effects of a global mixing at finite rate. By performing a perturbative analysis from the large mixing rate limit, we will show that, at least, one species has to go deterministically extinct for any species number $N \geq 3$ in the large size limit. We call this extinction occurring in the large size limit as deterministic extinction. The deterministic extinction is completely different from the extinction caused by stochasticity, where internal fluctuations as a result of a finite system size could cause the extinction of a species, in particular, when the species population size is low 28. It turns out that such deterministic extinction is key to understand why the relationship between the lattice models and the LV-equation for cyclic ecosystems was elusive.

\section{MODEL}

Let us denote a site on a ring (a one-dimensional lattice with the periodic boundary condition) by an index $i \in$ $\{1, \ldots, L\}$, and a species on site $i$ by $\sigma_{i} \in \mathcal{S} \equiv\{1, \ldots, N\}$ with $N \geq 3$. Each species has one prey and one predator through a cyclic competition as shown in Fig. 1. Explicitly, we consider a continuous time Markov process, where the species at site $i$ with $\sigma_{i}=\alpha$ invades the nearest neighbor sites $j \in\{i-1, i+1\}$ with rate $1 / 2$ only if $\sigma_{j}=\alpha_{+}$where $\alpha_{ \pm} \equiv \alpha \pm 1$ modulo $N$, making $\sigma_{j}=\alpha$ after invasion. In addition to this invading process, we introduce the exchange of two individual species on a uniformly chosen random pair of two sites $i, j$ at rate $v / 2$ per one site as global mixing [29]: Namely, $\left(\sigma_{i}, \sigma_{j}\right)=(\alpha, \beta)$ with $\alpha, \beta \in \mathcal{S}$ is updated as $\left(\sigma_{i}, \sigma_{j}\right)=(\beta, \alpha)$ after mixing. Note that this mixing process does not change the population density of each species in the system [30]. The initial probability of finding each species $\alpha$ on site $i$ is assumed to be determined by $Q(\alpha)$, independent of site $i$, namely with $\sum_{\alpha \in \mathcal{S}} Q(\alpha)=1$.

We define the $n$-point probability $P_{\left\{\alpha_{k}\right\}_{k=1}^{n}}(t)$ as the probability of a randomly chosen segment of $n$ sites containing the sequence of species $\left\{\alpha_{k}\right\}_{k=1}^{n}$, in this order from the left to the right, with $\alpha_{k} \in \mathcal{S}$ for any integer $k$, $1 \leq k \leq n$ at time $t$. The time evolution of the one-point probability can be determined by taking into account all possible changes of species in invasion processes at pairs of adjacent sites. Then, the one-point probability is de- scribed by the following two-point probabilities

$$
\dot{P}_{\alpha}=\frac{1}{2}\left(P_{\alpha, \alpha_{+}}+P_{\alpha_{+}, \alpha}-P_{\alpha_{-}, \alpha}-P_{\alpha, \alpha_{-}}\right) .
$$

In the limit of $L \rightarrow \infty$ and $v \rightarrow \infty$, one can easily find that this becomes the LV equation (1). Thus, in general, the evolution equation of $(n-1)$-point probability is exactly described by only the $n$-point probability [24]. Note that Eq. 22 does not explicitly depend on the mixing rate $v$ due to the conservation of the populations under mixing, while explicit contributions from mixing arise in $\dot{P}_{\left\{\alpha_{k}\right\}_{k=1}^{n}}$ for any $n \geq 2$.

\section{RESULTS}

\section{Perturbative approach to deterministic extinction}

We employ a perturbation approach from the infinite mixing rate where the limit of $L \rightarrow \infty$ is taken first. In order to obtain a closed set of evolution equations, it is key to realize the following relation for any integer $n \geq 2$ :

$$
P_{\left\{\alpha_{k}\right\}_{k=1}^{n}}=\frac{P_{\left\{\alpha_{k}\right\}_{k=1}^{n-1}} P_{\left\{\alpha_{k}\right\}_{k=2}^{n}}}{P_{\left\{\alpha_{k}\right\}_{k=2}^{n-1}}}+\mathcal{O}\left(v^{-1-2(n-2)}\right),
$$

where $P_{\left\{\alpha_{k}\right\}_{k=2}^{1}} \equiv 1$ and $\mathcal{O}\left(x^{a}\right)$ has a finite real number $C$ independent of $x$ such that $\mathcal{O}\left(x^{a}\right) / x^{a} \leq C$ when $x \ll 1$. The term $O\left(v^{-1}\right)$ comes from the fact that in order for $\alpha_{1}$ to influence $\alpha_{N}$, initially species $\alpha_{1}$ at the most left site has to invade $\alpha_{2}$ before mixing to make predator $\alpha_{1}$ away. The probability that such an event occurs is $\mathcal{O}(1 / v)$. Then, the rest of the term $\mathcal{O}\left(v^{-2(n-2)}\right)$ comes from the fact that $\alpha_{1}$ must succeed to invade $\alpha_{n}$ through the segment of $(n-2)$-sites to influence $\alpha_{n}$ at the most right site by invading all species in between and $\alpha_{1}$ at the most left site must not be affected by the mixing. The probability that such an event occur is $\mathcal{O}\left(v^{-2}\right)$ at each invasion, leading to $\mathcal{O}\left(v^{-2(n-2)}\right)$ totally. Note that Eq. (3) with $n=3$ ignoring $\mathcal{O}\left(v^{-1-2(n-2)}\right)$ was used as (Kirkwood) pair approximation in order to analyze the case of $v=0$ [22 24].

Using Eq. (3) with $n=3$ and ignoring the term of $\mathcal{O}\left(v^{-3}\right)$, we obtain the following equation for any $\alpha, \beta \in$ $\mathcal{S}:$

$$
\begin{gathered}
\dot{P}_{\alpha, \beta}=\frac{1}{2}\left(-\delta_{\beta, \alpha_{+}} \frac{P_{\alpha, \beta}}{P_{\beta}} \sum_{\gamma \in \mathcal{S} \backslash\{\beta\}} P_{\beta, \gamma}-\frac{P_{\alpha, \beta} P_{\beta, \beta_{-}}}{P_{\beta}}\right. \\
-\frac{P_{\alpha_{-}, \alpha} P_{\alpha, \beta}}{P_{\alpha}}-\delta_{\alpha, \beta_{+}} \frac{P_{\alpha, \beta}}{P_{\alpha}} \sum_{\gamma \in \mathcal{S} \backslash\{\alpha\}} P_{\gamma, \alpha} \\
+\frac{P_{\alpha, \alpha_{+}} P_{\alpha_{+}, \beta}}{P_{\alpha_{+}}}\left(1-\delta_{\beta, \alpha_{+}}\right)+\frac{P_{\alpha_{,} \beta_{+}} P_{\beta_{+}, \beta}}{P_{\beta_{+}}}\left(1-\delta_{\alpha, \beta_{+}}\right) \\
\left.+\delta_{\alpha, \beta}\left(P_{\alpha, \alpha_{+}}+P_{\beta_{+}, \beta}\right)\right) \\
+2 v\left(P_{\alpha} P_{\beta}-P_{\alpha, \beta}\right),
\end{gathered}
$$

where $\delta_{a, b}$ is the Kronecker delta function and $\mathcal{S} \backslash\{\alpha\}$ denotes the set obtained by excluding $\alpha$ from set $\mathcal{S}$. Thus, 
Eq. (2) and Eq. (4) give a closed set of equations, allowing us to calculate exactly $P_{\alpha}$ and $P_{\alpha, \beta}$ within the errors of $\mathcal{O}\left(v^{-3}\right)$. It should be noted that, precisely speaking, we should add that the reliability of the calculation holds up to only the time regime $t \ll \mathcal{O}\left(v^{3}\right)$ for sufficiently large values of $v$ because of the possible accumulation of error terms.

We now move on to introduce an indicator:

$$
\eta(t) \equiv \min _{\alpha \in \mathcal{S}} P_{\alpha}(t)
$$

in order to discuss the extinction of, at least, one species, which is defined as $\eta \rightarrow 0$ as $t \rightarrow \infty$. Indeed, instead of directly focusing on $\eta$, it turns out that it is convenient to consider a quantity $E \in[0,1]$ representing the intrinsic cyclic symmetry:

$$
E(t) \equiv N^{N} \prod_{\alpha \in \mathcal{S}} P_{\alpha}(t)
$$

because $E=0$ corresponds to $\eta=0$ and thus one may conclude that extinction occurs if $E \rightarrow 0$ as $t \rightarrow \infty$. One can easily find that $E$ is a conserved quantity in the limit of $v \rightarrow \infty$ by directly analyzing the LV equation (1) [1, 16, 17.

Let us estimate $\dot{E}$ within $\mathcal{O}\left(v^{-2}\right)$ by using Eq. (3) with $n=2$ for $P_{\alpha, \beta}$ in the following way:

$$
P_{\alpha, \beta}=P_{\alpha} P_{\beta}+\Delta_{\alpha, \beta} v^{-1}+\mathcal{O}\left(v^{-2}\right),
$$

where the order of $\Delta_{\alpha, \beta}$ is $\mathcal{O}\left(v^{0}\right)$ due to Eq. (3). By substituting Eq. (7) into Eq. (4), one obtains the explicit expression of $\Delta_{\alpha, \alpha_{+}}$as

$$
\Delta_{\alpha, \alpha_{+}}=\frac{1}{4} P_{\alpha} P_{\alpha_{+}}\left(P_{\alpha_{-}}+P_{\alpha}-P_{\alpha_{+}}-P_{\alpha_{++}}-1\right),
$$

and relation $\Delta_{\alpha, \alpha_{+}}=\Delta_{\alpha_{+}, \alpha}$. Then, one may finally obtain

$$
\dot{E}=-\frac{1}{4} E \sum_{\alpha \in \mathcal{S}}\left(P_{\alpha_{-}}-P_{\alpha_{+}}\right)^{2} v^{-1}+\mathcal{O}\left(v^{-2}\right) .
$$

Assuming that this perturbation series has a nonzeroradius of convergence in terms of $v^{-1}$, let us elaborate on what Eq. 97 means in terms of extinction. Indeed, $\dot{E}$ is always non-positive and can be zero only if the state is extinct with $E=0$ or balanced with $P_{\alpha_{-}}=P_{\alpha_{+}}$for any $\alpha \in \mathcal{S}$. Further, $E$ takes the maximum value 1 only when the state is symmetric with $P_{\alpha}=1 / N$ for any $\alpha \in \mathcal{S}$. Note that in the case of odd species number, this symmetric condition is the only case to satisfy the balanced condition $P_{\alpha_{+}}=P_{\alpha_{-}}$. Therefore, we may conclude $E \rightarrow 0$ as $t \rightarrow \infty$ unless the initial condition satisfies $Q\left(\alpha_{-}\right)=Q\left(\alpha_{+}\right)$for any $\alpha \in \mathcal{S}$.

In the case of even species number, there are asymmetric balanced states with $P_{\alpha_{-}}=P_{\alpha_{+}}>0$ and $P_{\alpha} \neq P_{\alpha_{+}}$ for any $\alpha \in \mathcal{S}$ where $E \notin\{1,0\}$. Therefore, such nontrivial asymmetric balanced points might affect the trajectories of states. In order to clarify this point, we consider Lotka-Volterra equation (2) combined with Eq. (7) and Eq. (8), which leads to an evolution equation (perturbative-LV equation) closed by only one-point probabilities. Indeed, asymmetric balanced points in the perturbative-LV equation are fixed points, which can be simply characterized as a set of $\left(q_{1}, q_{2}\right)$ where we put $P_{\alpha}=q_{1} \leq P_{\alpha_{+}}=q_{2}$ for a odd species number $\alpha$ without loss of generality. Therefore, the Jacobian matrix at the asymmetric balanced points has a certain circulant property. Namely, the Jacobian matrix can be constructed by only three different $2 \times 2$ matrices as entries and this Jacobian matrix is circulant in terms of those entries. Note that the analytical expressions of eigenvalues for any circulant matrix are well-known independent of the matrix sizes 31.

Therefore, using a standard method for usual circulant matrices with taking into account such a quasi-circulant property, one may obtain analytical expression of those $N$ eigenvalues $\left\{\lambda_{k}^{ \pm}\right\}_{k=1}^{N / 2}$ for any even species number $N$ as follows:

$$
\begin{aligned}
\lambda_{k}^{ \pm} & =2 \epsilon q_{1} q_{2}\left(1-\Re\left(\zeta^{k}\right)\right) \\
& \pm \boldsymbol{i} \sqrt{2 q_{1} q_{2}(1-\epsilon)^{2}\left(1-\Re\left(\zeta^{k}\right)\right)}+\mathcal{O}\left(\epsilon^{2}\right)
\end{aligned}
$$

where $\boldsymbol{i}$ is the imaginary unit, $\epsilon \equiv v^{-1} / 4, \quad \zeta=$ $\exp (\boldsymbol{i} 4 \pi / N), k$ is integer such that $1 \leq k \leq N / 2$, and $\Re(A)$ is the real part of a complex number $A$. An important point is that $\Re\left(\lambda_{k}^{ \pm}\right)>0$ for $k \neq N / 2$ because $\left(1-\Re\left(\zeta^{k}\right)\right) \geq 0$ and the equality holds only when $k=N / 2$. This zero eigenvalue is a trivial consequence of $\sum_{\alpha \in \mathcal{S}} P_{\alpha}=1$. This means that the asymmetric balanced fixed points with $P_{\alpha}=q_{1} \gg \epsilon$ are unstable. Note that in the case of $\epsilon=0$ and $q_{1}=q_{2}$, one may directly derive $\lambda_{k}^{ \pm}= \pm \boldsymbol{i}(2 / N) \sin (2 \pi k / N)$ from Eq. 10), which is consistent to the eigenvalue of the LV equation (1) at the fixed point with the symmetric condition for general species number $N$ [1].

Summarizing the above results, within the perturbation calculation in terms of $v^{-1}$, we conclude that for any species numbers $N \geq 3, E \rightarrow 0$ as $t \rightarrow \infty$ unless the initial condition satisfies $Q(\alpha)=Q\left(\alpha_{++}\right)$and $Q\left(\alpha_{-}\right)=Q\left(\alpha_{+}\right)$for any $\alpha \in \mathcal{S}$. This leads to $\eta \rightarrow 0$ as $t \rightarrow \infty$ under the same condition. Note that under the discussion until here, the fixed point with $P_{\alpha}=1$ and $P_{\beta}=0$ for any $\beta \neq \alpha$ is one candidate of the state with $E=0$. However, the eigenvalues at this fixed point in the limit of $\epsilon=0$ take only three values from $\{1,-1,0\}$ for general species number $N$, meaning that this fixed point with $E=0$ is still unstable for sufficient small values of $\epsilon$. Thus, next, we examine the different types of trajectories that appear as $E$ approaches 0 . 

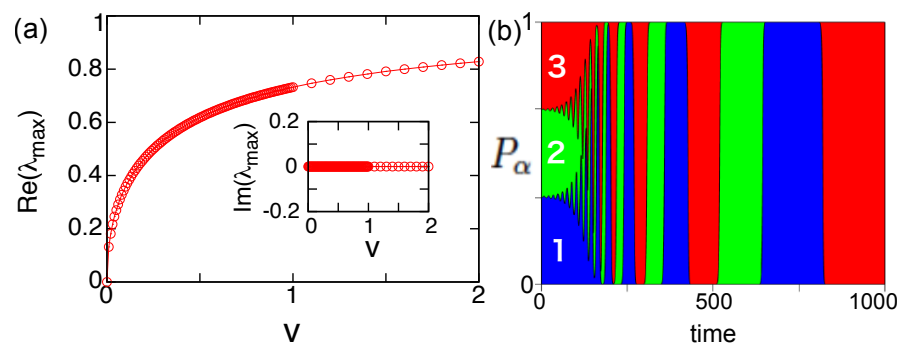

FIG. 2: (color online) (a) The maximum real part of the eigenvalues $\lambda$ at the extinction fixed points for $N=3$. The imaginary part is shown in the inset. (b) $P_{\alpha}(t)$ computed by Eqs. (2) and (4) for $v=1$ from the symmetric initial condition with a perturbation.

\section{Numerical analysis of extinction trajectories and bifurcation}

We next discuss through which trajectory the system approaches the extinction $\eta \rightarrow 0$, in particular, by employing the linear stability analysis of Eq. (2) and (4) at both fixed points $P_{\alpha} P_{\alpha_{+}}=0$ for any $\alpha \in \mathcal{S}$ and $P_{\alpha}=1 / N$ for any $\alpha \in \mathcal{S}$, with $\dot{P}_{\alpha}=0, \dot{P}_{\alpha, \beta}=0$ for any $\alpha, \beta \in \mathcal{S}$. For later convenience, we call the former fixed point where at least one species has zero population as an extinction fixed point, and the latter fixed point as a symmetric fixed point. Note that one can easily reduce the number of variables from $N+N^{2}$ to $N^{2}-N$ for any species number $N$ by using conservation laws such as $\sum_{\alpha \in \mathcal{S}} P_{\alpha}=1$. Therefore, we actually deal with $\left(N^{2}-N\right) \times\left(N^{2}-N\right)$ Jacobian matrix. In contrast to the perturbative Lotka-Volterra equation derived in the previous section, the evolution equations closed by onepoint and two point probabilities have more information about correct trajectories, especially for small values of $v$. However, such a linear stability analysis for rather small values of $v$ should be referred to as an approximation, nevertheless, it would give us insightful information as found below.

We computed $P_{\alpha, \beta}$ at the symmetric fixed points by solving Eqs. (2) and (4) numerically from the initial condition where $P_{\alpha}=1 / N, P_{\alpha, \beta}=\delta_{\alpha, \beta} / N$. By using this initial condition with no invasions, one can avoid coarsening effects causing slow relaxations. At the extinction fixed points, one can obtain the exact relation $P_{\alpha, \beta}=P_{\alpha} P_{\beta}$. Hereafter, we call the eigenvalue with the largest real part among all the eigenvalues as the dominant eigenvalue and the corresponding eigenvector as the dominant eigenvector. If the real part of the dominant eigenvalue is positive, the dominant eigenvector is also referred to as the most unstable eigenvector. Let us discuss the cases of $N=3, N=4$, and also $5 \leq N \leq 8$ in this order below.

In the case of 3 -species competition $(N=3)$, there are three extinction fixed points where two species have zero populations, namely $P_{\alpha}=1, P_{\alpha_{+}}=P_{\alpha_{-}}=0$ for any $\alpha \in \mathcal{S}$. The linear stability analysis indicates that the dominant eigenvalue is positive at any finite $v$ as shown in Fig. 2 a. Thus, the extinction fixed points turn out to be saddles. Therefore, one consistent way to realize the trajectory to extinction satisfying $\eta \rightarrow 0$ is a heteroclinic cycle connecting such unstable extinction fixed points. As shown in Fig. 2p, by solving numerically Eq. 22 and Eq. (4) from the initial conditions close to the symmetric fixed point, we indeed observed such heteroclinic cycles. This kind of trajectories was also observed by looking at the species densities in MC simulations at rather small $v$. This implies that mixing always causes the deterministic extinction for $N=3$ even with small $v$, leading to the heteroclinic cycle.

In the case of 4 -species competition, there are two connected regions parameterized by a real number $p \in[0,1]$, consisting of extinction fixed points corresponding to $P_{\alpha}=p, P_{\alpha_{++}}=1-p$, and $P_{\alpha_{+}}=P_{\alpha_{-}}=0$ for $\alpha=1,2$ where survival of two species is allowed. As shown in Fig. 3 a , we found that there is a region in the phase space, close to $p=1 / 2$, where only the dominant eigenvalue is zero, meaning that all the rest eigenvalues have negative real parts. Since the direction along the connected extinction fixed points in the phase space is obviously neutrally stable, corresponding the zero eigenvalue, these extinction fixed points around $p=1 / 2$ compose an attractor. As $v$ is increased, the extinction fixed points for smaller $p \leq 1 / 2$ lose its local stability, leading that this extinction attractor disappears for any $p$ in the limit of $v \rightarrow \infty$. Also, the stability analysis of the symmetric fixed point revealed that as $v$ is decreased from a sufficiently large value, at $v=v_{\mathrm{c}} \approx 0.19$, the most unstable eigenvector at the symmetric fixed point changes from an oscillatory mode to a non-oscillatory mode (Fig. 3 b). Indeed, the numerical solutions of Eq. (2) and Eq. (4) from the initial conditions close to the symmetric fixed point provide more information about what happen near $v=v_{\mathrm{c}}$ as follows. For $v=1>v_{\mathrm{c}}$, the trajectories seem to end up with a heteroclinic cycle as shown in Fig. 3d, implying that the basin of the extinction attractor around $p=1 / 2$ is far from the trajectories starting from the symmetric fixed point toward the heteroclinic cycle. However, for $v=0.02<v_{\mathrm{c}}$, trajectories mostly fall into the extinction attractor as shown in Fig. 3r. Thus, at the bifurcation point, from the viewpoint of the phase space, the newly appearing trajectory starting from the symmetric fixed point seems to collide with a boundary of the basin of the extinction attractor; thus, this bifurcation is a global bifurcation 32. We also observed these two different types of trajectories by MC simulation as shown in Fig. 33(e-f).

We performed the linear stability analysis at the symmetric fixed point for also larger $N$. In the cases of $N=5,6,7,8$, we also found switching of the most unstable eigenvector at certain values of $v$. In the case of 

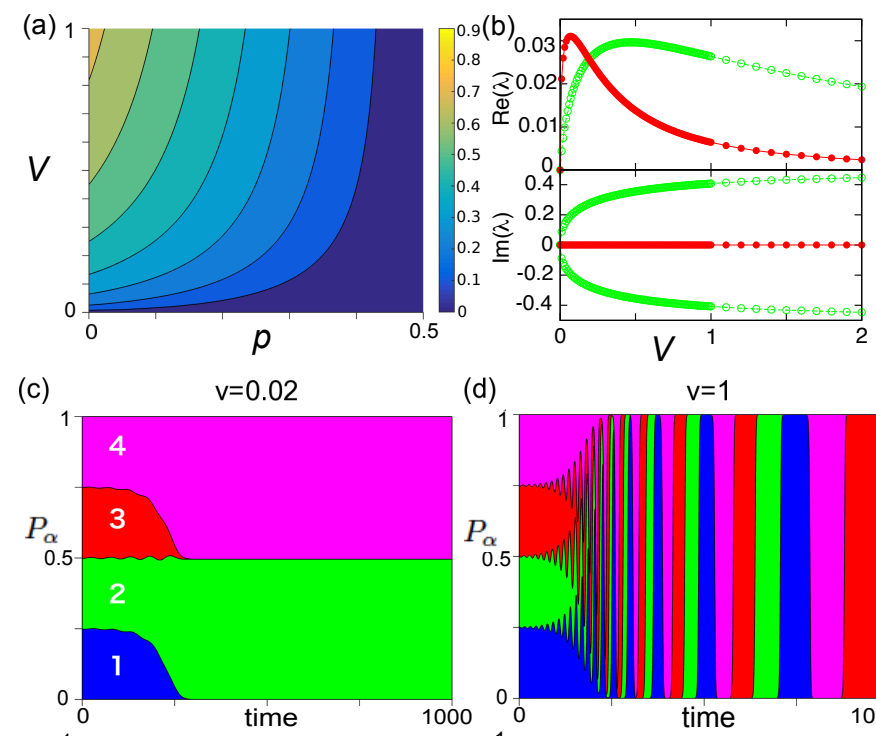

(d)
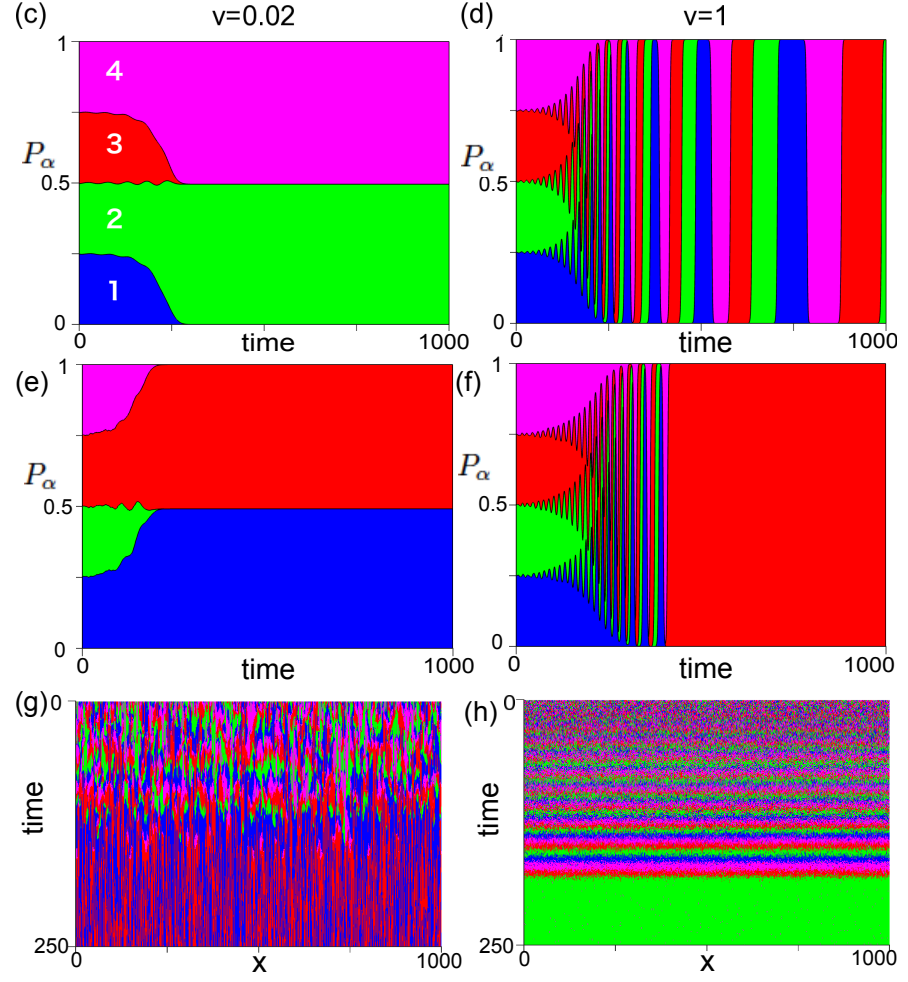

FIG. 3: (color online) (a) The maximum real part of eigenvalues at the extinction fixed point for $N=4$ as a function of $p$. (b) Eigenvalues $\lambda$ at the symmetric fixed point with $N=4$. The top panel shows the two eigenvalues with the largest and the second largest real part, and the bottom panel shows their respective imaginary parts. (c) $P_{\alpha}(t)$ computed by Eqs. 22 and (4) with $N=4$ from the symmetric initial condition with a perturbation for $v=0.02$. (d) $P_{\alpha}(t)$ computed by Eqs. (2) and (4) with $N=4$ from the symmetric initial condition with a perturbation for $v=1$. (e-f) MC simulations: The species densities with $L=9000(\mathrm{e}, \mathrm{f})$ and the spatiotemporal plot with $L=1000(\mathrm{~g}, \mathrm{~h})$ for $v=0.02(\mathrm{e}, \mathrm{g})$ and $v=1(\mathrm{f}, \mathrm{h})$, where $x$ is site number.

even $N$, as observed in 4 species, there is an eigenvector with zero imaginary part for any finite $v$, that becomes the most unstable eigenvector through a bifurcation as $v$ is decreased. In the case of odd $N$, the dominant eigenvector has non-zero imaginary part for any finite $v$. Importantly, in both cases of even and odd species number $N \geq 4$, we numerically observed that a global bifurcation occurs as $v$ is decreased from a sufficiently large value. Below the bifurcation, the system converges to an extinc- tion fixed point where several species survive as observed also in 4-species case. Specifically, in typical numerical experiments from the symmetric initial condition with a perturbation, just $\lfloor N / 2\rfloor$ species have nonzero populations at the reached extinction fixed points in the case of species number $N$. Note that for $N=3$, we did not find any switching of the most unstable eigenvector. Finally, for general $N$ as far as we have studied, the real part of the dominant eigenvalue is non-negative and approaches zero only in the limit of both $v \rightarrow 0$ and $v \rightarrow \infty$. Thus, from the viewpoint of this instability of the symmetric fixed point, the two limits of $v \rightarrow 0$ and $v \rightarrow \infty$ are rather singular.

\section{CONCLUDING REMARKS}

In this paper, we have studied a cyclically competing species model on a ring with a global mixing. With a perturbative analysis combined with numerical methods, we have clarified that the deterministic extinction occurs for any species number $N \geq 3$ at rather general mixing rate $v$ and its singular appearance from the two limits of $v \rightarrow 0$ and $v \rightarrow \infty$ is a key to bridge the gap between two descriptions of the LV equation and lattice models.

It would be natural to ask how the dimension of a lattice affects the dynamics of the system with invasion rate $1 / 2 d$. Indeed, in general, the deterministic extinction at sufficiently large mixing rates is expected to remain in higher dimensions because for such large mixing rates, the system would behave as if invasion rate was unchanged compared to that in one dimension. Nevertheless, since it is also expected that some dynamical behaviors different from those in one dimension occur for rather small mixing rates in higher dimension, it is intriguing to perform the detailed numerical and analytical studies in higher dimensions. The current analysis for one dimension could be generalized to that for higher dimensions. As a key point for such further studies, in order to compute the dynamics of the two-point probabilities as an exact perturbation in terms of $v^{-1}$, it seems to be necessary to consider $2 d+1$ point probabilities which comes from one site and its $2 d$ neighbor sites, instead of three point probabilities.

The global mixing studied in this paper is not only of theoretical interest. For example, a bacterial system consisting of toxin-producing, sensitive, and immune bacteria strains can form a cyclic competition and the mixing process in a liquid culture could be effectively global because of a vigorous mixing [9, 10. The present results in this paper imply that the effects of deterministic extinction could be rather widely observed in experiments because of the difficulties to realize precisely a well-mixed condition in reality. In experiments, we expect that the effects from deterministic extinction are observed when each species density is sufficiently far from 
zero, and eventually one species goes extinct because of intrinsic stochasticity in the finite-size system when the minimum species density gets close to zero compared to the strength of the stochasticity.

Lastly, let us mention the universality of the deterministic extinction in the presence of a mixing process in terms of different spatial descriptions. Indeed, the deterministic extinction by mixing similar to the behavior studied in this paper has been observed in a lattice model of 3-species cyclic competition under a flow at fast mixing [27, and also in the framework of the continuous limit described by a partial differential equation of populations under a turbulent convective flow at fast mixing 33. Such observations of deterministic extinction in different spatial descriptions imply the universality of deterministic extinction in cyclic systems with fast mixing, to some extent. Thus, we hope that the obtained results in this paper could provide a fundamental viewpoint to develop our understanding of mixing-induced deterministic extinction in more general ecosystems.

\section{ACKNOWLEDGMENTS}

The authors thank E. Frey for useful discussions on the topic related to Ref. 33 and also Y. Ishitsuka for telling us about the eigenvalues of a matrix with a quasicirculant property. This work is supported by the Danish National Research Foundation.

* Electronic address: mitarai@nbi.dk

$\dagger$ Electronic address: ohta.hiroki.6c@kyoto-u.ac.jp

[1] J. Hofbauer and K. Sigmund, Evolutionary Games and Population Dynamics, Cambridge University Press, (1998).

[2] G. Szabó and G. Fath, Phys. Rep. 446, 97 (2007).

[3] A. Szolnoki, M. Mobilia, L.-L. Jiang, B. Szczesny, A. M. Rucklidge, and M. Perc, J. R. Soc. Interface 11, 20140735 (2014).

[4] Y. Itoh, Proc. Japan Acad. 47, 854 (1971).

[5] R. M. May and W. J. Leonard, SIAM J. Appl. Math. 29, 243, (1975).

[6] K. Tainaka, J. Phys. Soc. Jpn. 57, 2588 (1988).

[7] M. Bramson and D. Griffeath, Ann. Prob. 17, 26 (1989).

[8] R. Durrett and S. Levin, Theor. Popul. Biol. 53, 30 (1998).
[9] T. L. Czárán, R. F. Hoekstra, and L. Pagie, Proc. Natl. Acad. Sci. 99, 786 (2002).

[10] B. Kerr, M. A. Riley, M. W. Feldman, and B. J. M. Bohannan, Nature 418, 171 (2002).

[11] J. Mathiesen, N. Mitarai, K. Sneppen, and A. Trusina, Phys. Rev. Lett. 107, 188101 (2011).

[12] Z. Frentz, S. Kuehn, and S. Leibler, Phys. Rev. X 5, 041014 (2015).

[13] K. Sato, N. Yoshida, and N. Konno, Appl. Math. Comput. 126, 225 (2002).

[14] Y. Itoh, Prog. Thor. Phys. 78, 507 (1987).

[15] O. Bogoyavlensky, J. Math. Phys. 50, 053517 (2009).

[16] C. H. Durney, S. O. Case, M. Pleimling, and R. K. P. Zia, Phys. Rev. E 83, 051108 (2011).

[17] J. Knebel, T. Kruger, M. F. Weber, and E. Frey, Phys. Rev. Lett. 110, 168106 (2013).

[18] J. O. Haerter, N. Mitarai, and K. Sneppen, Plos Comput. Biol. 12, e1004727 (2016).

[19] R. Fisch, Ann. Prob. 20, 1528 (1992).

[20] L. Frachebourg, P. L. Krapivsky, and E. Ben-Naim, Phys. Rev. E 54, 6186 (1996).

[21] L. Frachebourg, P. L. Krapivsky, and E. Ben-Naim, Phys. Rev. Lett. 77, 2125 (1996).

[22] K. Tainaka, J. Thor. Biol. 166, 91 (1994).

[23] K. Tainaka, Phys. Rev. E 50, 3401 (1994).

[24] L. Frachebourg and P. L. Krapivsky, J. Phys. A: Math. Gen. 31, L287 (1998).

[25] T. Reichenbach, M. Mobilia, and E. Frey, Phys. Rev. Lett. 99, 238105 (2007).

[26] T. Reichenbach, M. Mobilia, and E. Frey, Nature 448, 1046 (2007).

[27] G. Károlyi, Z. Neufeld, and I. Scheuring, J. Theor. Biol. 236, 12 (2005).

[28] T. Reichenbach, M. Mobilia, and E. Frey, Phys. Rev. E 74, 051907 (2006).

[29] A. R. Akhmetzhanov, L. Worden, and J. Dushoff, Phys. Rev. E 88, 012816 (2013).

[30] As a mixing process, one can consider a copy-paste process where a species at a randomly chosen site invades a species at another randomly chosen site with rate $v$. In this case, the main results in this paper are unchanged although each species density is often not conserved by each mixing process.

[31] P. J. Davis, Circulant Matrices, 2nd ed. AMS Chelsea Publishing, (1994)

[32] J. Guckenheimer and P. Holmes, Nonlinear Oscillations, Dynamical Systems, and Bifurcations of Vector Fields, Springer-Verlag, (1983).

[33] D. Grošelj, F. Jenko, and E. Frey, Phys. Rev. E 91, 033009 (2015). 